\title{
ON DECAY ESTIMATES OF SOLUTIONS FOR SOME DEGENERATE NONLINEAR KIRCHHOFF STRINGS WITH DISSIPATION
}

\author{
Kosuke Ono \\ Department of Mathematical Sciences \\ The University of Tokushima \\ Tokushima 770-8502, JAPAN
}

Abstract: We consider the initial-boundary value problem for the degenerate nonlinear dissipative wave equation of Kirchhoff type:

$$
u_{t t}-\left(\int_{0}^{1}\left|u_{x}(x, t)\right|^{2} d x\right)^{\gamma} u_{x x}+u_{t}+f(u)=0
$$

where $f(u)$ is like as $|u|^{p} u$. If the initial energy is appropriately small and $\gamma \geq 1$ and $p>2 \gamma$, then we obtain some optimal time decay estimates of the solution $u=u(t)$.

AMS Subject Classification: 35L80, 35B40, 35L20

Key Words: dissipative wave equations, degenerate, Kirchhoff strings, decay estimates

\section{Introduction}

In this paper we investigate the decay properties of solutions to the initialboundary value problem for the following degenerate nonlinear dissipative wave equation of Kirchhoff type: for $0<x<1,0<t<\infty$, 


$$
\begin{aligned}
& u_{t t}-\left(\int_{0}^{1}\left|u_{x}(x, t)\right|^{2} d x\right)^{\gamma} u_{x x}+u_{t}+f(u)=0, \\
& u(x, 0)=u_{0}(x), \quad u_{t}(x, 0)=u_{1}(x), \quad 0<x<1, \\
& u(0, t)=u(1, t)=0, \quad 0<t<\infty,
\end{aligned}
$$

where $u=u(x, t)$ is an unknown real value function and $\gamma \geq 1$ and $f(u)$ is a $C^{1}$-function satisfying

$$
\begin{aligned}
& 0 \leq F(u) \leq k_{0} f(u) u, \quad F(u) \equiv \int_{0}^{u} f(\eta) d \eta, \\
& |f(u)| \leq k_{1}|u|^{p+1}, \quad 0 \leq f^{\prime}(u) \leq k_{2}|u|^{p}, \quad p>0
\end{aligned}
$$

with positive constants $k_{0}, k_{1}, k_{2}>0$.

Equation (1) describes small amplitude vibrations of an elastic string (see Kirchhoff [6] for the original equation).

Through this paper, we will use the following energy $E\left(u, u_{t}\right)$ and functional $H\left(u, u_{t}\right)$ associated with (1):

$$
\begin{aligned}
& E\left(u, u_{t}\right) \equiv \frac{1}{2}\left\|u_{t}\right\|^{2}+\frac{1}{2(\gamma+1)}\left\|u_{x}\right\|^{2(\gamma+1)}+\int_{\Omega} F(u) d x, \\
& H\left(u, u_{t}\right) \equiv \frac{\left\|u_{x t}\right\|^{2}}{\left\|u_{x}\right\|^{2 \gamma}}+\left\|u_{x x}\right\|^{2},
\end{aligned}
$$

where the symbol $\|\cdot\|$ is the usual norm of $L^{2}=L^{2}(\Omega)$ with $\Omega=(0,1)$. We often denote $E(t) \equiv E\left(u(t), u_{t}(t)\right)$ and $H(t) \equiv H\left(u(t), u_{t}(t)\right)$ for simplicity. In particular, we will use the following notations related with the initial data $\left\{u_{0}, u_{1}\right\}$ :

$$
\begin{aligned}
& E(0) \equiv \frac{1}{2}\left\|u_{1}\right\|^{2}+\frac{1}{2(\gamma+1)}\left\|u_{0, x}\right\|^{2(\gamma+1)}+\int_{\Omega} F\left(u_{0}\right) d x, \\
& H(0) \equiv \frac{\left\|u_{1, x}\right\|^{2}}{\left\|u_{0, x}\right\|^{2 \gamma}}+\left\|u_{0, x x}\right\|^{2} .
\end{aligned}
$$

When the initial data belong to usual Sobolev spaces, Arosio \& Garabaldi [1] have studied on the unique local weak solutions for the Kirchhoff type wave equations (also see [2], [3] and the references cited therein).

In the non-degenerate case, Hosoya and Yamada [5] have proved global existence theorem and they have derived that the energy has an exponential decay estimate under some small data conditions. 
On the other hand, in the degenerate case and $f(u) \equiv 0$, many authors have studied the global existence theorem and the decay estimates of solutions (see [9], [11] and the references cited therein).

In the previous paper [10], we have shown the existence of the unique global solution of (1) and we have derived the decay estimate of the energy $E(t)$ associated with (1).

Theorem 1.1. Suppose that

$$
\gamma \geq 1 \text { and } p+1>2 \gamma
$$

and the initial data $\left\{u_{0}, u_{1}\right\}$ belong to $H^{2} \cap H_{0}^{1} \times H_{0}^{1}$ with $u_{0} \neq 0$ and $\left\{u_{0}, u_{1}\right\}$ are appropriately small in the sense of (19). Then, the problem (1) admits a unique global solution $u(t)$ in the class $C^{0}\left([0, \infty) ; H^{2} \cap H_{0}^{1}\right) \cap C^{1}\left([0, \infty) ; H_{0}^{1}\right) \cap$ $C^{2}\left([0, \infty) ; L^{2}\right),\left\|u_{x}(t)\right\| \neq 0$, and the energy $E(t)$ satisfies $E(t) \leq C(1+t)^{-1-\frac{1}{\gamma}}$, that is,

$$
\begin{aligned}
& 0<\left\|u_{x}(t)\right\|^{2} \leq C(1+t)^{-\frac{1}{\gamma}}, \\
& \left\|u_{t}(t)\right\|^{2} \leq C(1+t)^{-1-\frac{1}{\gamma}} \quad \text { for } \quad t \geq 0,
\end{aligned}
$$

where $C$ is some positive constant.

The purpose of this paper is to derive the decay estimates which are more detailed the previous estimates (8) and (9). In particular, we will show the lower decay estimates of $\left\|u_{x}(t)\right\|$ and $\left\|u_{x x}(t)\right\|$, and we will improve the decay estimate of $\left\|u_{t}(t)\right\|$. In order to obtain these estimates, we use the new identity (33) associated with the $H^{2}$-norm of the solution $u(t)$ and the function $\alpha(t)$ given by (29).

Our main result is as follows.

Theorem 1.2. Suppose that the assumption of Theorem 1.1 is fulfilled and

$$
\gamma \geq 1 \text { and } p>2 \gamma
$$

and the initial energy $E(0)$ is appropriately small in the sense of (37). Then, the solution $u(t)$ of (1) satisfies that

$$
\begin{aligned}
& C^{-1}(1+t)^{-\frac{1}{\gamma}} \leq\left\|u_{x}(t)\right\|^{2},\left\|u_{x x}(t)\right\|^{2} \leq C(1+t)^{-\frac{1}{\gamma}}, \\
& \left\|u_{t}(t)\right\|^{2} \leq C(1+t)^{-2-\frac{1}{\gamma}} \quad \text { for } \quad t \geq 0,
\end{aligned}
$$

where $C$ is some positive constant. 
The notations we use in this paper are standard. The symbol $(\cdot, \cdot)$ means the inner product in $L^{2}=L^{2}(\Omega), \Omega=(0,1)$, or sometimes duality between the space $X$ and its dual $X^{\prime}$. We denote the Sobolev-Poincaré constant by $c_{*}$, that is, $\|v\|_{p} \leq c_{*}\left\|v_{x}\right\|$ for $1 \leq p \leq \infty$, where $\|\cdot\|_{p}$ is the usual $L^{p}$-norm $\left(\|\cdot\|=\|\cdot\|_{2}\right.$ if $p=2$ ). We denote $(a)^{+}=\max \{0, a\}$.

\section{Preliminaries}

By standard arguments, we have the following local existence theorem (see [1], [3], [10] and the references cited therein). We omit the proof.

Proposition 2.1. Suppose that the initial data $\left\{u_{0}, u_{1}\right\}$ belong to $H^{2} \cap$ $H_{0}^{1} \times H_{0}^{1}$ and $u_{0} \neq 0$. Then, the problem (1) admits a unique local solution $u(t)$ in the class $C\left([0, T) ; H^{2} \cap H_{0}^{1}\right) \cap C^{1}\left([0, T) ; H_{0}^{1}\right) \cap C^{2}\left([0, T) ; L^{2}\right)$ for some $T \equiv$ $T\left(\left\|u_{0}\right\|_{H^{2}},\left\|u_{1}\right\|_{H^{1}}\right)>0$. Moreover, if $\left\|u_{x}(t)\right\|>0$ and $\|u(t)\|_{H^{2}}+\left\|u_{t}(t)\right\|_{H^{1}}<\infty$ for $t \geq 0$, then we can take $T=\infty$.

In what follows, we denote $\left\|u_{x}(t)\right\|^{2}$ by $M(t)$ for simplicity. From the definition (3) of the energy $E(t) \equiv E\left(u(t), u_{t}(t)\right)$, it is easy to see that

$$
M(t) \equiv\left\|u_{x}(t)\right\|^{2} \leq(2(\gamma+1) E(t))^{\frac{1}{\gamma+1}} .
$$

By simple calculation, we see the energy $E(t)$ has the energy identity

$$
\frac{d}{d t} E(t)+\left\|u_{t}(t)\right\|^{2}=0
$$

or

$$
E(t)+\int_{0}^{t}\left\|u_{t}(s)\right\|^{2} d s=E(0) .
$$

Indeed, multiplying (1) by $u_{t}$ and integrating over $\Omega$ or $\Omega \times(0, t)$, we obtain (14) or (15). Moreover, applying energy method together with the Nakao's inequality (see citeNa78 and [8]), we have the following decay estimate of the energy $E(t)$ (see [7] and [10] for the proof).

Proposition 2.2. Let $u(t)$ be a solution of (1). Then the energy $E(t)$ satisfies

$$
E(t) \leq\left(E(0)^{-\frac{\gamma}{\gamma+1}}+d_{1}^{-1}\left(E(0)^{\frac{\gamma}{\gamma+1}}+1\right)^{-1}(t-1)^{+}\right)^{-\frac{\gamma+1}{\gamma}}
$$

for $t \geq 0$, where $d_{1}$ is some positive constant. 
Moreover, we see immediately that the inequality (13) and the energy decay (16) yield the following estimates.

Corollary 2.3. Let $u(t)$ be a solution of $(1)$ and $E(0) \leq 1$.

(i) If $q>\gamma$, then

$$
\int_{0}^{t} M(s)^{q} d s \leq d_{2} E(0)^{\frac{q-\gamma}{\gamma+1}}
$$

(ii) If $q>\gamma / 2$, then

$$
\int_{0}^{t}(1+s)^{-\frac{1}{2}} M(s)^{q} d s \leq d_{3} E(0)^{\frac{1}{\gamma+1}\left(q-\frac{\gamma}{2}\right)},
$$

where $d_{2}$ and $d_{3}$ are some positive constants.

\section{Global Existence}

In this section we will prove Theorem 1.1.

Proposition 3.1. Let $u(t)$ be a solution and $M(t) \equiv\left\|u_{x}(t)\right\|^{2}>0$. Suppose that $\gamma \geq 1$ and $p+1>2 \gamma$ and $u_{0} \neq 0$ and

$$
I(0) \equiv 2(2(\gamma+1) E(0))^{\frac{\gamma-1}{2(\gamma+1)}}\left(H(0)+d_{4} E(0)^{\frac{p+1-2 \gamma}{\gamma+1}}\right)^{\frac{1}{2}}<\frac{2}{\gamma+2},
$$

where $d_{4}$ is some positive constant given by (27). Then, it holds that

$$
\begin{aligned}
& H(t) \leq H(0)+d_{3} E(0)^{\frac{p+1-2 \gamma}{\gamma+1}}, \\
& \frac{\left|M^{\prime}(t)\right|}{M(t)} \leq I(0)<\frac{2}{\gamma+2} .
\end{aligned}
$$

Proof. Multiplying (1) by $\left(-2 u_{x x t} / M(t)^{\gamma}\right)$ and integrating it over $\Omega$, we have

$$
\begin{aligned}
& \frac{d}{d t} H(t)+2\left(1+\frac{\gamma}{2} \frac{M^{\prime}(t)}{M(t)}\right) \frac{\left\|u_{x t}(t)\right\|^{2}}{M(t)^{\gamma}}=-\frac{2}{M(t)^{\gamma}}\left(f(u), u_{x x t}\right) \\
& \leq \frac{2 k_{2}}{M(t)^{\gamma}}\|u(t)\|_{\infty}^{p}\left\|u_{x}(t)\right\|\left\|u_{x t}(t)\right\| \leq 2 k_{2} c_{*}^{p}\left(M(t)^{p+1-\gamma} \frac{\left\|u_{x t}(t)\right\|^{2}}{M(t)^{\gamma}}\right)^{\frac{1}{2}} .
\end{aligned}
$$


We observe from (4) and (15) that

$$
\begin{aligned}
\frac{\left|M^{\prime}(t)\right|}{M(t)} & \leq 2 \frac{\left\|u_{x t}(t)\right\|}{\left\|u_{x}(t)\right\|}=2\left(M(t)^{\gamma-1} \frac{\left\|u_{x t}(t)\right\|^{2}}{M(t)^{\gamma}}\right)^{\frac{1}{2}} \\
& \leq 2(2(\gamma+1) E(0))^{\frac{\gamma-1}{2(\gamma+1)}} H(t)^{\frac{1}{2}} .
\end{aligned}
$$

In what follows, we may assume that $E(0) \leq 1$.

Under the assumption (19), putting

$$
T \equiv \sup \left\{t \mid 2(2(\gamma+1) E(0))^{\frac{\gamma-1}{2(\gamma+1)}} H(s)^{\frac{1}{2}}<\frac{2}{\gamma+2}, 0 \leq s<t\right\},
$$

then we see that $T>0$.

If $T<\infty$, then

$$
2(2(\gamma+1) E(0))^{\frac{\gamma-1}{2(\gamma+1)}} H(T)^{\frac{1}{2}}=\frac{2}{\gamma+2} .
$$

For $0 \leq t \leq T$, we observe from (23)-(26) that

$$
1+\frac{\gamma}{2} \frac{\left|M^{\prime}(t)\right|}{M(t)} \geq 1-\frac{\gamma}{2} \frac{2}{\gamma+2}=\frac{2}{\gamma+2}
$$

and from (22) that

$$
\frac{d}{d t} H(t)+\frac{4}{\gamma+2} \frac{\left\|u_{x t}(t)\right\|^{2}}{M(t)^{\gamma}} \leq 2 k_{2} c_{*}^{p}\left(M(t)^{p+1-\gamma} \frac{\left\|u_{x t}(t)\right\|^{2}}{M(t)^{\gamma}}\right)^{\frac{1}{2}},
$$

and from the Young inequality that

$$
\frac{d}{d t} H(t) \leq \frac{\gamma+2}{4}\left(k_{2} c_{*}^{p}\right)^{2} M(t)^{p+1-\gamma}
$$

Moreover, if $p+1>2 \gamma$, we have from (17) in Corollary 2.3 that

$$
H(t) \leq H(0)+d_{4} E(0)^{\frac{p+1-2 \gamma}{\gamma+1}}, \quad d_{4}=\frac{\gamma+2}{4}\left(k_{2} c_{*}^{p}\right)^{2} d_{2} .
$$

Then, we have from (19) that

$$
2(2(\gamma+1) E(0))^{\frac{\gamma-1}{2(\gamma+1)}} H(t)^{\frac{1}{2}} \leq I(0)<\frac{2}{\gamma+2} \quad \text { for } \quad 0 \leq t<T,
$$

which is a contradiction to (26), and hence, we see that $T=\infty$, and we conclude that (27) and (28) hold true for $t \geq 0$. 
Proof of Theorem 1.1. By $u_{0} \neq 0$, we see $M(0)>0$. If there exists $T>0$ such that $M(t)>0$ for $0 \leq t<T$ and $M(T)=0$, then since $H(t) \leq C<\infty$ by (20), we have that $\lim _{t \rightarrow T}\left\|u_{x t}(t)\right\|=0$, and hence, we see that $\left\{u(T), u_{t}(T)\right\}=$ $\{0,0\}$.

On the other hand, by the backward uniqueness to (1) with $\left\{u(T), u_{t}(T)\right\}=$ $\{0,0\}$ (see [9] and [10]), we observe that $u \equiv 0$ on $[0, T]$, which is a contradiction to the assumption $u_{0} \neq 0$. Thus, we conclude that $M(t)>0$ for $t \geq 0$, and moreover, from Proposition 3.1 we obtain the a-priori estimate $\|u(t)\|_{H^{2}}+$ $\left\|u_{t}(t)\right\|_{H^{1}} \leq C<\infty$ for $t \geq 0$. Therefore, the local solution $u(t)$ of (1) in the sense of Proposition 2.1 can be continued globally in time. Also, from Proposition 2.2 we obtain the decay estimates (7) and (8) for $t \geq 0$.

\section{Proof of Theorem 1.2}

In this section, let $u(t)$ be the global solution of (1) given by Theorem 1.1. In order to get the sharp decay estimates of $\|u(t)\|_{H^{2}}$ and $\left\|u_{t}(t)\right\|$, we use the following function $\alpha(t)$ defined by

$$
\alpha(t) \equiv \sup _{0 \leq s \leq t}\left\{(1+s) \frac{\left\|u_{t}(s)\right\|^{2}}{M(s)^{\gamma+1}}\right\} .
$$

Proposition 4.1. Suppose that $p>2 \gamma$. Then, it holds that

$$
\frac{\left\|u_{x x}(t)\right\|^{2}}{M(t)} \leq G(t) \leq 2 G(0)+d_{5} E(0)^{\frac{p-\gamma}{\gamma+1}} \alpha(t),
$$

where $d_{5}$ is some positive constant given by (35) and

$$
\begin{aligned}
& G(t) \equiv \frac{\left\|u_{x x}(t)\right\|^{2}}{M(t)}+\frac{2}{M(t)^{\gamma+2}}\left((f(u))_{x}, u_{x}\right)+Q(t) \quad(\geq 0), \\
& Q(t) \equiv \frac{1}{M(t)^{\gamma+2}}\left(M(t)\left\|u_{x x}(t)\right\|^{2}-\left(\frac{1}{2} M^{\prime}(t)\right)^{2}\right) \quad(\geq 0) .
\end{aligned}
$$


Proof. Since we observe from (1) that

$$
\begin{aligned}
& M(t)^{\gamma} \frac{d}{d t}\left\|u_{x x}(t)\right\|^{2}=2\left(M(t)^{\gamma} u_{x x}, u_{x x t}\right) \\
& \quad=-2\left\|u_{x t}(t)\right\|^{2}-2\left(u_{x t t}, u_{x t}\right)+2 \frac{d}{d t}\left(f(u), u_{x x}\right)-2\left((f(u))_{t}, u_{x x}\right), \\
& M(t)^{\gamma}\left\|u_{x x}(t)\right\|^{2}=2\left(M(t)^{\gamma} u_{x x}, u_{x x}\right) \\
& \quad=-\frac{1}{2} M^{\prime}(t)+\left\|u_{x t}(t)\right\|^{2}-\frac{1}{2} M^{\prime \prime}(t)+\left(f(u), u_{x x}\right),
\end{aligned}
$$

we have

$$
\frac{d}{d t}\left(\frac{\left\|u_{x x}(t)\right\|^{2}}{M(t)}+\frac{2}{M(t)^{\gamma+1}}\left((f(u))_{x}, u_{x}\right)\right)=-2 Q(t)-R(t)+S(t),
$$

where $Q(t)$ is given by $(32)$ and

$$
\begin{aligned}
R(t) & \equiv \frac{1}{M(t)^{\gamma+2}}\left(M(t) \frac{d}{d t}\left\|u_{x t}(t)\right\|^{2}+M^{\prime}(t)\left(\left\|u_{x t}(t)\right\|^{2}-\frac{1}{2} M^{\prime \prime}(t)\right)\right), \\
S(t) & \equiv \frac{1}{M(t)^{\gamma+2}}\left((2 \gamma+1) M^{\prime}(t)\left(f(u), u_{x x}\right)-2 M(t)\left((f(u))_{t}, u_{x x}\right)\right) .
\end{aligned}
$$

On the other hand, we have

$$
\begin{aligned}
\frac{d}{d t} Q(t) & =-(\gamma+2) \frac{M^{\prime}(t)}{M(t)} Q(t) \\
& +\frac{1}{M(t)^{\gamma+2}}\left(M(t) \frac{d}{d t}\left\|u_{x t}(t)\right\|^{2}+M^{\prime}(t)\left\|u_{x t}(t)\right\|^{2}-\frac{1}{2} M^{\prime}(t) M^{\prime \prime}(t)\right) \\
& =-(\gamma+2) \frac{M^{\prime}(t)}{M(t)} Q(t)+R(t) .
\end{aligned}
$$

Adding (33) to (34), we have

$$
\frac{d}{d t} G(t)=-2\left(1+\frac{\gamma+2}{2} \frac{M^{\prime}(t)}{M(t)}\right) Q(t)+S(t)
$$

Moreover, since we observe from (21) that

$$
1+\frac{\gamma+2}{2} \frac{\left|M^{\prime}(t)\right|}{M(t)} \geq 0, Q(t) \geq 0,\left((f(u))_{x}, u_{x}\right)=\int_{\Omega} f^{\prime}(u)\left|u_{x}\right|^{2} d x \geq 0,
$$


and

$$
\begin{aligned}
|S(t)| \leq & \frac{2(2 \gamma+1) k_{2}}{M(t)^{\gamma+2}}\left\|u_{t}(t)\right\|\left\|u_{x x}(t)\right\|\|u(t)\|_{\infty}^{p}\left\|u_{x}(t)\right\|^{2} \\
& +\frac{2 k_{2}}{M(t)^{\gamma+1}}\|u(t)\|_{\infty}^{p}\left\|u_{t}(t)\right\|\left\|u_{x x}(t)\right\| \\
\leq & c_{2}\left(\frac{\left\|u_{t}(t)\right\|^{2}}{M(t)^{\gamma+1}} \frac{\left\|u_{x x}(t)\right\|^{2}}{M(t)} M(t)^{p-\gamma}\right)^{\frac{1}{2}}
\end{aligned}
$$

with $c_{2}=4(\gamma+1) k_{2} c_{*}^{p}$, we have

$$
\frac{d}{d t} G(t) \leq c_{2}\left(\frac{\left\|u_{t}(t)\right\|^{2}}{M(t)^{\gamma+1}} G(t) M(t)^{p-\gamma}\right)^{\frac{1}{2}}
$$

or

$$
2 \frac{d}{d t} G(t)^{\frac{1}{2}} \leq c_{2}\left((1+t)^{-1} M(t)^{p-\gamma}\right)^{\frac{1}{2}}\left((1+t) \frac{\left\|u_{t}(t)\right\|^{2}}{M(t)^{\gamma+1}}\right)^{\frac{1}{2}}
$$

Thus, we obtain from (29) and (18) that if $p>2 \gamma$,

$$
\begin{aligned}
2 G(t)^{\frac{1}{2}} & \leq 2 G(0)^{\frac{1}{2}}+c_{2} \alpha(t)^{\frac{1}{2}} \int_{0}^{t}(1+s)^{-\frac{1}{2}} M(s)^{\frac{p-\gamma}{2}} d s \\
& \leq 2 G(0)^{\frac{1}{2}}+c_{2} d_{2} E(0)^{\frac{1}{\gamma+1}\left(\frac{p}{2}-\gamma\right)} \alpha(t)^{\frac{1}{2}},
\end{aligned}
$$

and hence,

$$
G(t) \leq 2 G(0)+d_{5} E(0)^{\frac{p-2 \gamma}{\gamma+1}} \alpha(t), \quad d_{5}=\frac{c_{2}^{2} d_{2}^{2}}{2}
$$

which implies the desired estimate (30).

Proposition 4.2. Suppose that $p>2 \gamma$ and $E(0)$ is appropriate small like as (37). Then, it holds that

$$
\frac{\left\|u_{t}(t)\right\|^{2}}{M(t)^{\gamma+1}} \leq C(1+t)^{-1}, \quad\left\|u_{t}(t)\right\|^{2} \leq C(1+t)^{-2-\frac{1}{\gamma}} .
$$

Proof. Multiplying (1) by $\left(2 u_{t} / M(t)^{\gamma+1}\right)$ and integrating it over $\Omega$, we 
have

$$
\begin{aligned}
\frac{d}{d t} & \frac{\left\|u_{t}(t)\right\|^{2}}{M(t)^{\gamma+1}}+2\left(1+\frac{\gamma+1}{2} \frac{M^{\prime}(t)}{M(t)}\right) \frac{\left\|u_{t}(t)\right\|^{2}}{M(t)^{\gamma+1}} \\
& =-\frac{M^{\prime}(t)}{M(t)}-\frac{2}{M(t)^{\gamma+1}}\left(f(u), u_{t}\right) \\
& \leq 2 \frac{\left\|u_{t}(t)\right\|}{M(t)^{\frac{\gamma+1}{2}}} \frac{\left\|u_{x x}(t)\right\|}{M(t)^{\frac{1}{2}}} M(t)^{\frac{\gamma}{2}}+2 c_{*}^{p+1} k_{1} \frac{\left\|u_{t}(t)\right\|}{M(t)^{\frac{\gamma+1}{2}}} M(t)^{\frac{p-\gamma}{2}}
\end{aligned}
$$

and from (21) that

$$
\begin{aligned}
& \frac{d}{d t} \frac{\left\|u_{t}(t)\right\|^{2}}{M(t)^{\gamma+1}}+\frac{2}{\gamma+2} \frac{\left\|u_{t}(t)\right\|^{2}}{M(t)^{\gamma+1}} \\
& \quad \leq 2 \frac{\left\|u_{t}(t)\right\|}{M(t)^{\frac{\gamma+1}{2}}}\left(\frac{\left\|u_{x x}(t)\right\|}{M(t)^{\frac{1}{2}}}+c_{*}^{p+1} k_{1} M(t)^{\frac{p-2 \gamma}{2}}\right) M(t)^{\frac{\gamma}{2}},
\end{aligned}
$$

and from the Young inequality and (15) and (30) that

$$
\begin{aligned}
& \frac{d}{d t} \frac{\left\|u_{t}(t)\right\|^{2}}{M(t)^{\gamma+1}}+b \frac{\left\|u_{t}(t)\right\|^{2}}{M(t)^{\gamma+1}} \\
& \quad \leq 2(\gamma+2)\left(\frac{\left\|u_{x x}(t)\right\|^{2}}{M(t)}+c_{*}^{2(p+1)} k_{1}^{2} M(t)^{p-2 \gamma}\right) M(t)^{\gamma} \\
& \quad \leq\left(c_{3}+2(\gamma+2) d_{5} E(0)^{\frac{p-2 \gamma}{\gamma+1}} \alpha(t)\right) M(t)^{\gamma}
\end{aligned}
$$

with $b=1 /(\gamma+2)$ and $c_{3}=2(\gamma+2)\left(2 G(0)+c_{*}^{2(p+1)} k_{1}^{2}(2(\gamma+1) E(0))^{\frac{p-2 \gamma}{\gamma+1}}\right)$, where we used (15) and (30) at the last inequality. We observe that

$$
\begin{aligned}
& \int_{0}^{t} e^{-b(t-s)} M(s)^{\gamma} d s=\left(\int_{0}^{t / 2}+\int_{t / 2}^{t}\right) e^{-b(t-s)} M(s)^{\gamma} d s \\
& \quad \leq e^{-\frac{b}{2} t}(2(\gamma+1) E(0))^{\frac{\gamma}{\gamma+1}} \frac{t}{2}+\frac{1}{b}\left(2(\gamma+1) E\left(\frac{t}{2}\right)\right)^{\frac{\gamma}{\gamma+1}} \\
& \quad \leq d_{6}(1+t)^{-1}
\end{aligned}
$$

where $d_{6}$ is some positive constant independent of $E(0)$ when $E(0) \leq 1$. Thus, we have

$$
\frac{\left\|u_{t}(t)\right\|^{2}}{M(t)^{\gamma+1}} \leq \frac{\left\|u_{1}\right\|^{2}}{M(0)^{\gamma+1}} e^{-b t}+d_{6}\left(c_{3}+2(\gamma+2) d_{5} E(0)^{\frac{p-2 \gamma}{\gamma+1}} \alpha(t)\right)(1+t)^{-1}
$$


and

$$
\alpha(t) \leq c_{4} \frac{\left\|u_{1}\right\|^{2}}{M(0)^{\gamma+1}}+d_{6}\left(c_{3}+2(\gamma+2) d_{5} E(0)^{\frac{p-2 \gamma}{\gamma+1}} \alpha(t)\right)
$$

with $c_{4}=\sup _{t \geq 0}(1+t) e^{-b t}$. If $E(0)$ is small like as

$$
2(\gamma+2) d_{5} d_{6} E(0)^{\frac{p-2 \gamma}{\gamma+1}}<1
$$

then we obtain

$$
\alpha(t) \leq C \quad \text { or } \quad \frac{\left\|u_{t}(t)\right\|^{2}}{M(t)^{\gamma+1}} \leq C(1+t)^{-1}
$$

which gives the desired estimate (36) with (8).

As a corollary of Proposition 4.1 and Proposition 4.2, we obtain the following estimate.

Proposition 4.3. Under the assumption of Proposition 4.2, it holds that

$$
\frac{\left\|u_{x x}(t)\right\|^{2}}{M(t)} \leq C, \quad\left\|u_{x x}(t)\right\|^{2} \leq C(1+t)^{-\frac{1}{\gamma}} .
$$

We will derive the lower decay estimate of the function $M(t)$.

Proposition 4.4. Under the assumption of Proposition 4.2, it holds that

$$
M(t) \equiv\left\|u_{x}(t)\right\|^{2} \geq C^{\prime}(1+t)^{-\frac{1}{\gamma}}
$$

with some positive constant $C^{\prime}$.

Proof. Multiplying (1) by $\left(2 u_{t} / M(t)^{\gamma+2}\right)$ and integrating it over $\Omega$, we have

$$
\begin{aligned}
\frac{d}{d t}( & \left.\frac{\left\|u_{t}(t)\right\|^{2}}{M(t)^{\gamma+2}}+\frac{1}{M(t)}\right)+2\left(1+\frac{\gamma+2}{2} \frac{M^{\prime}(t)}{M(t)}\right) \frac{\left\|u_{t}(t)\right\|^{2}}{M(t)^{\gamma+2}} \\
= & -2 \frac{M^{\prime}(t)}{M(t)^{2}}-\frac{2}{M(t)^{\gamma+2}}\left(f(u), u_{t}\right) \\
\leq & 2\left(\frac{\left\|u_{t}(t)\right\|^{2}}{M(t)^{\gamma+2}}\right)^{\frac{1}{2}}\left(\frac{\left\|u_{x x}(t)\right\|^{2}}{M(t)} M(t)^{\gamma-1}\right)^{\frac{1}{2}} \\
& +2 c_{*}^{p+2}\left(\frac{\left\|u_{t}(t)\right\|^{2}}{M(t)^{\gamma+2}}\right)^{\frac{1}{2}}\left(M(t)^{p-2 \gamma} M(t)^{\gamma-1}\right)^{\frac{1}{2}}
\end{aligned}
$$


and from (21) and the Young inequality that

$$
\begin{aligned}
\frac{d}{d t}\left(\frac{\left\|u_{t}(t)\right\|^{2}}{M(t)^{\gamma+2}}+\frac{1}{M(t)}\right) & \leq C \frac{\left\|u_{x x}(t)\right\|^{2}}{M(t)} M(t)^{\gamma-1}+C M(t)^{p-2 \gamma} M(t)^{\gamma-1} \\
& \leq C M(t)^{\gamma-1} \leq C(1+t)^{-1+\frac{1}{\gamma}},
\end{aligned}
$$

where we used the estimates (16) and (38) at the last inequality.

Thus, we obtain

$$
\frac{\left\|u_{t}(t)\right\|^{2}}{M(t)^{\gamma+2}}+\frac{1}{M(t)} \leq C+C \int_{0}^{t}(1+s)^{-1+\frac{1}{\gamma}} d s \leq C(1+t)^{\frac{1}{\gamma}},
$$

and hence, we see $M(t) \geq C^{\prime}(1+t)^{-\frac{1}{\gamma}}$ for $t \geq 0$.

Proof of Theorem 1.2. The upper decay estimate (11) follows from (38) in Proposition 4.3. The lower decay estimate 11 follows from (39) in Proposition 4.4. The sharp decay estimate (12) follows from (36) in Proposition 4.2.

The proof of Theorem 1.2 is now completed.

\section{Acknowledgments}

This work was partially supported by Grant-in-Aid for Science Research (C) 21540186 of JSPS (Japan Society for the Promotion of Science).

\section{References}

[1] A. Arosio and S. Garavaldi, On the mildly degenerate Kirchhoff string, Math. Methods Appl. Sci., 14 (1991), 177-195.

[2] A. Arosio and S. Panizzi, On the well-posedness of the Kirchhoff string, Trans. Amer. Math. Soc., 348 (1996), 305-330.

[3] H.R. Crippa, On local solutions of some mildly degenerate hyperbolic equations, Nonlinear Analysis, 21 (1993), 565-574.

[4] R.W. Dickey, Infinite systems of nonlinear oscillation equations with linear damping, SIAM J. Appl. Math., 19 (1970), 208-214.

[5] M. Hosoya and Y. Yamada, On some nonlinear wave equations. II. Global existence and energy decay of solutions, J. Fac. Sci. Univ. Tokyo Sect. IA Math., 38 (1991), 239-250. 
[6] G. Kirchhoff, Vorlesungen über Mechanik, Teubner, Leipzig, (1883).

[7] M. Nakao, A difference inequality and its application to nonlinear evolution equations, J. Math. Soc. Japan, 30 (1978), 747-762.

[8] M. Nakao and K. Ono, Existence of global solutions to the Cauchy problem for the semilinear dissipative wave equations, Math. Z., 214 (1993), 325342.

[9] K. Nishihara and Y. Yamada, On global solutions of some degenerate quasilinear hyperbolic equations with dissipative terms, Funkcial. Ekvac., 33 (1990), 151-159.

[10] K. Ono, Global existence and decay properties of solutions for some mildly degenerate nonlinear dissipative Kirchhoff strings, Funkcial. Ekvac., 40 (1997), 255-270.

[11] K. Ono, On sharp decay estimates of solutions for mildly degenerate dissipative wave equations of Kirchhoff type, Math. Methods Appl. Sci., 34 (2011), 1339-1352. 
